\title{
ROSI and GEANT4 - A comparison in the context of high energy X-ray physics
}

\author{
Markus Kiunke ${ }^{\mathrm{a}}$, Carina Stritt ${ }^{\mathrm{b}, *}$, Richard Schielein ${ }^{\mathrm{a}}$, Frank Sukowski $^{\mathrm{d}}$, Astrid Hölzing ${ }^{\mathrm{d}}$, Simon Zabler ${ }^{\mathrm{a}, \mathrm{d}}$, Jürgen Hofmann $^{\mathrm{c}}$, \\ Alexander Flisch ${ }^{\mathrm{c}}$, Stefan Kasperl ${ }^{\mathrm{d}}$, Urs Sennhauser ${ }^{\mathrm{b}}$, Randolf Hankea,d \\ ${ }^{a}$ Chair of X-ray Microscopy, University Würzburg, Josef-Martin-Weg 63, 97074 Würzburg, Germany \\ ${ }^{b}$ Empa, Swiss Federal Laboratories for Material Science and Technology, Reliability Science and Technology, Überlandstrasse 129, 8600 Dübendorf, Switzerland \\ ${ }^{c}$ Empa, Swiss Federal Laboratories for Material Science and Technology,Center for X-ray Analytics, Überlandstrasse 129, 8600 Dübendorf, Switzerland \\ ${ }^{d}$ Fraunhofer Development Center for X-ray Technology EZRT; Flugplatzstr. 75, 90768 Fürth, Germany
}

\begin{abstract}
This work compares two popular MC simulation frameworks ROSI (Roentgen Simulation) and GEANT4 (Geometry and Tracking in its fourth version) in the context of X-ray physics. The comparison will be performed with the help of a parameter study considering energy, material and length variations. While the total deposited energy as well as the contribution of Compton scattering show a good accordance between all simulated configurations, all other physical effects exhibit large deviations in a comparison of data-sets. These discrepancies between simulations are shown to originate from the different cross sectional databases used in the frameworks, whereas the overall simulation mechanics seem to not have an influence on the agreement of the simulations. A scan over energy, length and material shows that the two parameters energy and material have a significant influence on the agreement of the simulation results, while the length parameter shows no noticeable influence on the deviations between the data-sets.
\end{abstract}

\section{Introduction}

In modern X-ray Computed Tomography (CT) setups the configuration of system and operating parameters have a significant effect on the quality of the measurement. Simulations are performed to optimise design and operating parameters. Due to the stochastic nature of the physical interactions in radiation transport, Monte Carlo (MC) methods are well suited. The goal of these simulations is to investigate performance and image quality of the CT system. The MC simulation process of photon transport allows not only the estimation of transmission images, but also enables the investigation of the influence of each physical interaction. Especially the impact of imaging artefacts related to physical effects, such as coherent and incoherent scattering, can be assessed quantitatively. Due to their high computation times, these simulations were not feasible in good statistics until recent years without the help of supercomputers. With the development of progressively powerful computers, the use of MC techniques, either in a pure form [1,2] or incorporated in a hybrid simulation [3, 4], has become a popular method for modelling of X-ray CT systems [5, 6, 7]. Current MC simulation frameworks offer a link to interaction cross section databases of all physical effects for many materials as well as geometries needed for modelling the CT setup.

The different kinds of simulation frameworks, while similar in concept, still show diverse implementations. Not only are they written in various programming languages, they might also differ concerning the simulation mechanics for particle transport. Hence, the comparability of simulation results originating from

\footnotetext{
${ }^{*}$ Corresponding author

Email address: Carina.Stritt@empa.ch (Carina Stritt)
}

different MC frameworks is not necessarily given. This means that the choice of the framework as well as other choices, such as the engine for random number generation as well as the choice of data-bases, if possible, can have a large effect on the outcome of a simulation. While the overall aim is to model and simulate reality as good as possible, it is hard to decide which simulation framework should be used in a given situation. This work will focus on the comparison of two simulation frameworks commonly used in the context of X-ray physics: Roentgen Simulation (ROSI) [8] and Geometry and Tracking in its fourth version (GEANT4) [9]. The two frameworks are similar, as they are both object-oriented MC codes. Nevertheless, they are based on different $\mathrm{C}++$ libraries and have different origins. Due to their wide-spread use in the same field of application $[10,11,12]$ it is interesting to see the agreement of the simulation results from both frameworks [13]. For this comparison, a simple setup has been employed and tested with multiple materials of different thicknesses as well as varying X-ray energies. The distribution of the resulting scattered photons and the information about the physical processes has been analysed.

\section{Monte Carlo Frameworks}

MC simulations are based on the repeated random sampling technique from a probability distribution of possible input values. They can be used when it is hard or even impossible to determine the result of a problem analytically. Because of the straight forward method of conducting a high number of randomly sampled experiments, MC simulations can incorporate a high number of degrees of freedom of a system. 


\subsection{ROSI}

The ROSI [8] simulation code was developed based on the objectoriented $\mathrm{C}++$ framework LSCAT-GISMO [14]. The C ++ class library Graphical Interface for Simulation and MC Code with Objects (GISMO) and the Low-Energy Photon-Scattering extension (LSCAT) [15] originated in the popular MC code EGS4 [16]. EGS4 is an established MC simulation code containing the algorithms for the interactions and the transport of electrons and photons in arbitrary geometry over a high energy range exceeding the $\mathrm{TeV}$ regime. In contrast to EGS4, which is written in MORTRAN [17], the simulation framework GISMO uses $\mathrm{C}++$ as a programming language. Later on the LSCAT extension of EGS4 was incorporated into GISMO to account for the interactions of low energy particles leading to the new version of GISMO called LSCAT-GISMO. While the GISMO framework was developed as a general MC simulation code, the ROSI simulation code was developed specifically with the application of X-ray imaging in mind. ROSI still uses the established EGS4 interaction algorithms. Moreover, it is possible to run the simulations in parallel in order to shorten simulation time.

\subsection{GEANT4}

The GEANT [9] series is a simulation framework developed at CERN. It uses MC methods to simulate particle dynamics and interactions. In contrast to its predecessors, which were written in FORTRAN, the newest version GEANT4 is based on object oriented $\mathrm{C}++$ programming. The version of GEANT4 used for the results in this work is 4.9.6.

GEANT4 is a MC simulation toolkit for single particle transport, which means that each particle is individually transported through the system in a step-wise manner. As a result of that, no interaction between two particles can be simulated. Via toolkits, the GEANT4 code allows the simulation of a wide variety of particles and physical interactions as well as geometries given either by a set of primitives or voxelized data. Not only the physical interactions themselves, but also the model, which describes the interactions can be chosen. The choice of the correct physics list and physics models depends on the specific application on hand and is crucial for the quality of the outcome of a simulation. In the case of X-ray imaging, discussed in this paper, the low energy electromagnetic databases given in GEANT4 will be used.

\section{Choice of databases}

The mechanics of the simulation of ROSI and GEANT are comparable, since both codes transport the particles through the system in a step-wise manner. As a result, the comparability of both MC Codes depends highly on the agreement of the databases for cross sections and material data. This is why in a first step it has to be made clear that the libraries used for the simulations in ROSI are within tolerable accordance with those used in GEANT4. The data in the libraries is composed of values taken from various formulas and databases. The next subsections quickly introduce both libraries and their corresponding databases.

\subsection{Low energy database in ROSI}

ROSI uses a low energy photon-scattering extension to EGS4 named LSCAT. It is a combination of analytical, semi-analytical and empirical corrections to the EGS4 code mainly to add Doppler broadening, fluorescence after a photoelectric effect or electron impact ionisation and scattering of polarised photons. The data from LSCAT is used for low energy scattering and the Livermore evaluated photon data library (EPDL97) [18], while NIST XCOM Data [19] for all remaining effects.

\subsection{Low energy databases in GEANT4}

The database for physics in GEANT4 can be chosen from a set of libraries. Others can be added as well, but then the user has to do the effort of implementing this new set of cross sections and other physical quantities manually. Here, the low energy electromagnetic physics are needed. Keep in mind that in GEANT4 everything below $1 \mathrm{GeV}$ counts as low energetic process. The two main choices for low energy physics in GEANT4 are the Livermore and the Penelope database. Both Penelope and Livermore cover a validity range from $250 \mathrm{eV}$ up to $1 \mathrm{GeV}$, which extends further down to $10 \mathrm{eV}$ with a slightly reduced accuracy. The Livermore model is based on the evaluated atomic data library (EADL) [20], evaluated electrons data library (EEDL) [21] and evaluated photons data library (EPDL97) [18]. The Penelope database is an approach that consists of a mixture of analytic, parameterized and database-driven models. Penelope improves the standard electromagnetic implementation of physics in GEANT4 up to an energy of $5 \mathrm{MeV}$. Above $5 \mathrm{MeV}$ the physics in Penelope are equal to those of the standard electromagnetic simulations. A description of the physical models used in both libraries can be found in table1.

\subsection{Choice of library}

Due to the fact, that both the GEANT4 library Livermore and the low energy database of ROSI are based on EPDL97 data we assume that the low energy electromagnetic library Livermore will show a better agreement with the cross sections from the ROSI database then the GEANT4 library Penelope. Moreover, both frameworks rely heavily on the NIST data sets for cross sections which promises good accordance between the two simulations. In order to not rule out the Penelope library completely, several additional tests comparing the ROSI simulations with simulations performed under the Penelope datasets as well as a cross comparison between both GEANT4 libraries will be performed.

\section{Methods}

\subsection{Simulation setup}

The simulations in both frameworks use the same setup, which can be seen in Fig.1. The setup is simulated without air as a standard medium. The X-ray source is a monoenergetic (adjustable) pencil beam situated in a distance of $200 \mathrm{~mm}$ with respect to the origin of the object. The rod-like object in the center of the simulation setup has a radius of $1 \mathrm{~mm}$. The length $\mathrm{d}$ is variable as well as the material of the object. An ideal 


\begin{tabular}{|l|l|l|}
\hline Livermore in GEANT4 & Penelope in GEANT4 & Compton interaction \\
\hline $\begin{array}{l}\text { Klein-Nishina cross section modified by } \\
\text { Hubbell form factor (EPDL97) }\end{array}$ & $\begin{array}{l}\text { Analytic parameterization (with atomic } \\
\text { binding and Doppler broadening) }\end{array}$ & $\begin{array}{l}\text { Klein-Nishina cross section (with } \\
\text { atomic binding and Doppler broaden- } \\
\text { ing) }\end{array}$ \\
\hline \multicolumn{3}{|c|}{ Rayleigh scattering } \\
\hline $\begin{array}{l}\text { Angular distribution from Rayleigh } \\
\text { formula, Hubbell form factor from } \\
\text { EPDL97 }\end{array}$ & Analytic parameterization & $\begin{array}{l}\text { The Rayleigh distribution from Storm } \\
\text { and Israel [22] and the form factor from } \\
\text { Hubbell and Øverbø [23] }\end{array}$ \\
\hline \multicolumn{3}{|c|}{ Photoelectric effect } \\
\hline $\begin{array}{l}\text { Cross sections from EPDL97, angular } \\
\text { distribution can be chosen }\end{array}$ & Sauter distribution & $\begin{array}{l}\text { Cross sections from Storm and Israel } \\
\text { [22] }\end{array}$ \\
\hline \multicolumn{3}{|c|}{ Pair Conversion } \\
\hline $\begin{array}{l}\text { Bethe-Heitler formula with Coulomb } \\
\text { correction }\end{array}$ & $\begin{array}{l}\text { Bethe-Heitler cross section with } \\
\text { Coulomb correction (semi-empirical } \\
\text { model) }\end{array}$ & $\begin{array}{l}\text { Cross sections from Motz et al. [24] and } \\
\text { empirical corrections from Butcher and } \\
\text { Messel [25]in Born approximation }\end{array}$ \\
\hline \multicolumn{3}{|c|}{ Bremstrahlung } \\
\hline Parameterization from EPDL97 & Total cross section calculated from data & $\begin{array}{l}\text { Cross sections from Koch and Motz [26] } \\
\text { and empirical corrections from Butcher } \\
\text { and Messel [25] in Born approximation }\end{array}$ \\
\hline $\begin{array}{l}\text { Parameterization through shell parame- } \\
\text { ters }\end{array}$ & Calculated analytically & Calculated analytically [27] \\
\hline
\end{tabular}

Tab. 1: Implemented physical models of the low energy electromagnetic interaction libraries of Livermore (emlivermore), Penelope (empenelope) as well as ROSI, for comparison.

spherical photon detector is placed around the center of the object. It has a radius of $1000 \mathrm{~mm}$ and is divided into equiangular bins of a quarter of a degree in both lateral and longitudinal direction. This amounts to a total of $1440 \times 720$ detection bins. There is no material assigned to the detector itself, which means that there is no interaction in the detector. Each detector bin records the energy of the incident photon. Furthermore by flagging photons originating from different physical interactions like photoelectric effect, (incoherent) Compton scattering, (coherent) Rayleigh scattering, electron-positron annihilation and bremsstrahlung in the object, the corresponding amounts of photons can be distinguished in the detector. The assignment of these physical interactions to the photons will be described in the next section. Furthermore this setup has already been presented in a previous paper [28], which shows scans over energy, length and material over the scattering angle in ROSI.

\subsection{Method of counting}

The assignment of the physical effects to the propagating photons is done via interaction trees. Children of particles that are created through the various interactions inherit all physical interactions their parent particles had undergone. However, the assignment of physical effects to the particle tracks is binary due to the sheer amount of data generated otherwise. This means, interactions which occurred multiple times, like a multiple Compton scattering event for example, will only be flagged as Compton scattering interaction, but the information about the number or the order of occurrences will not be considered. However, since a particle can originate from an interaction tree

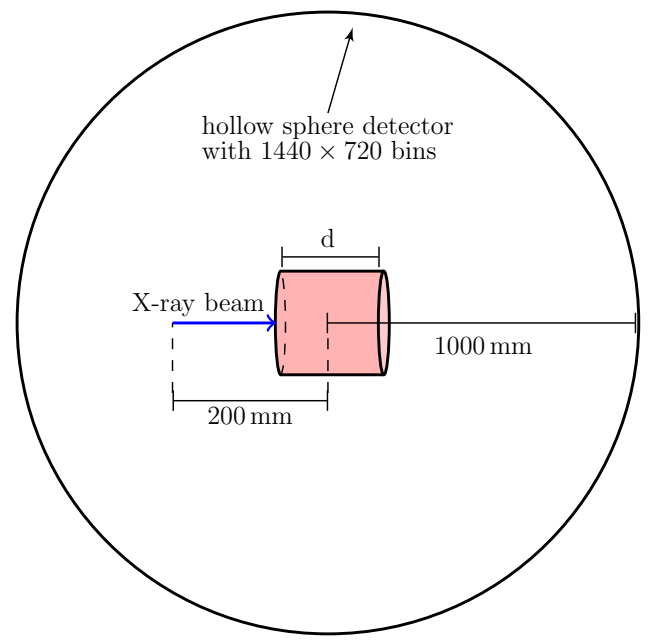

Fig. 1: Simulation setup. The X-ray source is situated at a distance of $200 \mathrm{~mm}$ with respect to the origin of the object. The object is rod-like with a radius of $1 \mathrm{~mm}$ and height $\mathrm{d}$, which can be varied. The detector has a spherical form with a radius of $1000 \mathrm{~mm}$. Detector resolution is $0.25^{\circ}$ which amounts to $1440 \times 720$ bins.

with several interactions, the deposited energy at the detector element can correspond to several physical interactions, too. This means that the sum of the radial representations of all physical effects will not add up to the total deposited energy ( $\left.E_{\text {total dep. }}\right)$. An example of this method of counting can be seen in Fig. 2. Each recorded photon at the detector will contribute to the plot of $E_{\text {total dep. }}$. This curve is taken as normalisation in the data 
analysis (refer to equation 1 ), which means that the integral over the total deposited energy of each detector element $\left(E_{\mathrm{tie}}\right)$ of the scattered radiation will yield a value of 1 .

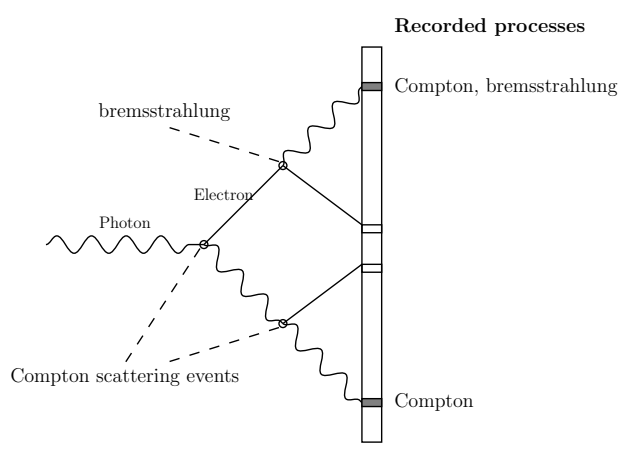

Fig. 2: Example of the counting method for an event with several interactions. Here, at first a Compton effect occurs and the resulting electron then decelerates under emission of a photon which is detected. It can be seen, that the deposited energy of the first recorded photon will be accounted for by both the radial representation of the Compton interaction and the representation corresponding to the effect of bremsstrahlung. The second recorded photon that can be seen in this sketch contributes only to the plot corresponding to the Compton interaction. The information that this photon did in fact undergo two Compton scattering events will not be considered in this case.

\subsection{Parameter configurations, evaluation and representation}

The simulated parameter configurations can be seen in Table 2. For the evaluation of each simulation the signal from the photons, which did not undergo any interaction, is removed. The data from the "hollow sphere" detector is then normalised with $E_{\text {tie }}$ and the two-dimensional angular distribution is combined into a polar representation. To account for the difference in counting rate per surface area the radial data is finally divided by the sine of the corresponding polar angle.

$$
\begin{aligned}
& R(\theta, p)=\frac{\int_{-180^{\circ}}^{180^{\circ}} D(\phi, \theta, p) d \phi}{E_{t i e} \cdot \sin (\theta)} \\
& D(\phi, \theta, p)=\text { deposited energy at detector element } \\
& (\phi, \theta) \text { for physical effect of type } p \\
& \text { with } p \in \text { Rayleigh, Compton, bremsstrahlung, } \\
& \text { photo effect, annihilation } \\
& E_{\text {total dep. }}(\phi, \theta)=\text { total deposited energy at detector } \\
& \text { element }(\phi, \theta) \\
& E_{\text {tie }}=\int_{0^{\circ}}^{180^{\circ}} \int_{-180^{\circ}}^{180^{\circ}} E_{\text {total dep. }}(\phi, \theta) d \phi d \theta
\end{aligned}
$$

From that we derive three graph representations of the data:

1. For a single database we show the logarithmic plot of the polar representation. According to symmetry only the angles from $0^{\circ}$ in forward direction up to $180^{\circ}$ for back scattering have to be taken into account.

$$
\mathcal{P}_{1}=\log \left(R\left(\theta=\left\{0^{\circ}, \cdots, 180^{\circ}\right\}, p\right)\right)
$$

The vertical line marks $90^{\circ}$, which means everything beyond that line can be considered as back scattered radiation.

2. For the comparison of the two data bases we show the ratio between the two separately normalised polar representations, which directly leads to a numeric factor describing the agreement of both results.

$$
\mathcal{P}_{2}=\frac{R_{\text {database } 1}\left(\theta=\left\{0^{\circ}, \cdots, 180^{\circ}\right\}, p\right)}{R_{\text {database } 2}\left(\theta=\left\{0^{\circ}, \cdots, 180^{\circ}\right\}, p\right)}
$$

The expected value is marked by a horizontal line at the factor of 1 .

3. An overview over the tendencies of the scattering contribution and their corresponding discrepancies in the data sets is given by the calculation of the mean value $\mu$ and the standard deviation std of each contribution for all data sets. The resulting data is plotted for 2 cases, where two of the three parameters (energy $E$, length $d$, material $M$ ) will be kept fixed.

$$
\begin{aligned}
\mathcal{P}_{3, E} & =\left\{\mu\left(\mathcal{P}_{2} \mid d, M\right), \operatorname{std}\left(\mathcal{P}_{2} \mid d, M\right)\right\} \\
\mathcal{P}_{3, d} & =\left\{\mu\left(\mathcal{P}_{2} \mid E, M\right), \operatorname{std}\left(\mathcal{P}_{2} \mid E, M\right)\right\}
\end{aligned}
$$

The mean value is an indicator for the systematic error between the compared simulations while the standard deviation gives an extent of the local errors.

\begin{tabular}{|l|c|l|}
\hline Parameter & Unit & Value \\
\hline Energy E & {$[\mathrm{MeV}]$} & $\{0.1,0.4,1.0,1.1,3.0,6.0\}$ \\
\hline Length $\mathrm{d}$ & {$[\mathrm{mm}]$} & $\{0.2,2.0,20.0,100.0\}$ \\
\hline Material $\mathrm{M}$ & & $\{\mathrm{Al}, \mathrm{PVC}, \mathrm{FeC}, \mathrm{Pb}\}$ \\
\hline
\end{tabular}

Tab. 2: List of parameter scans of the performed simulations.

\section{Results}

For each of the parameter configurations given in Table 2, a simulation with $10^{8}$ primary photons has been performed, as a result of a trade off between simulation time and statistical uncertainties. In the following, a representative case of the resulting polar distributions will be shown and discussed as well as a representative case for the ratio of the data sets. Finally, a parameter sweep over energy, length and material is analysed in order to reveal overall tendencies or trends in the parameter space.

\subsection{Representative study of a polar distribution}

This first analysis discusses the exemplary study of the case of a lead rod. It has a length of $100.0 \mathrm{~mm}$ and the simulation was realised using a primary photon beam at an energy of $3.0 \mathrm{MeV}$. Figure $3 \mathrm{a}$ and Figure $3 \mathrm{~b}$ show the simulation results of both 


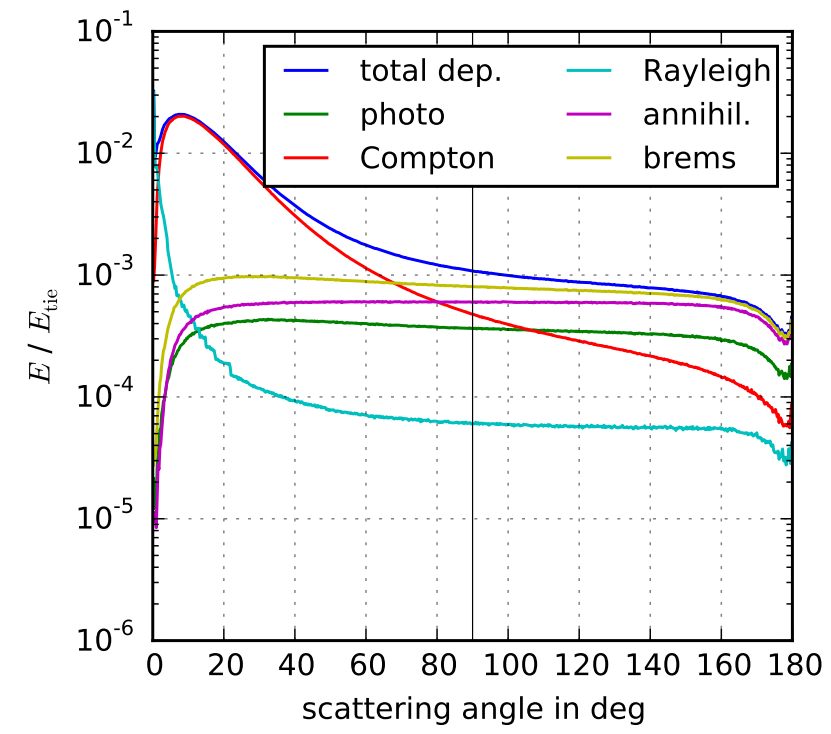

(a) The energy divided by $E_{\text {tie }}$ vs. the scattering angle in degree for $10^{8}$ primary photons simulated in ROSI

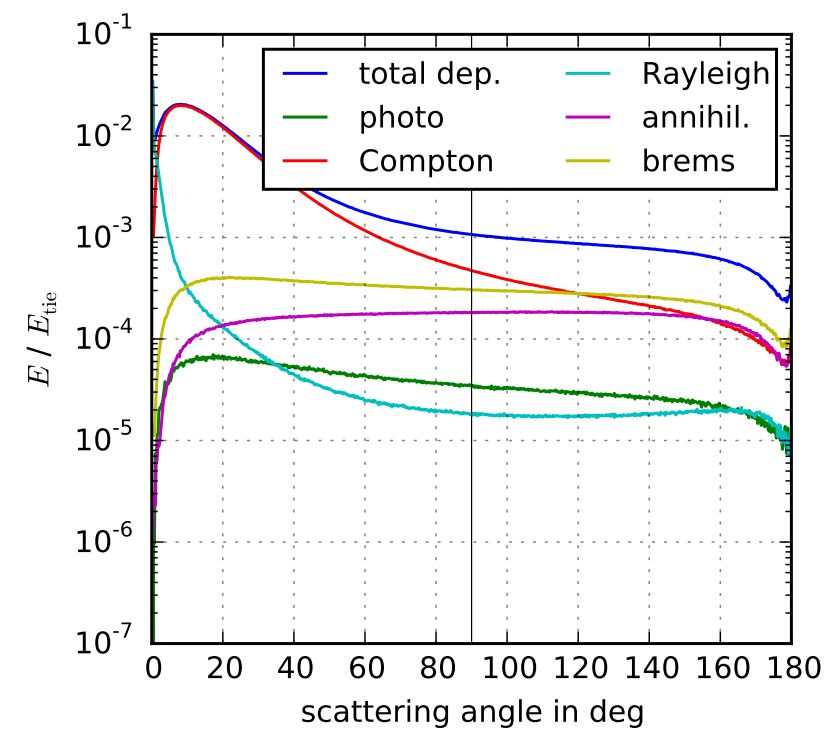

(b) The energy divided by $E_{\text {tie }}$ vs. the scattering angle in degree for $10^{8}$ primary photons simulated in GEANT4 in the emlivermore configuration

Fig. 3: The normalised energy vs. the scattering angle is shown for the exemplary case of a $100.0 \mathrm{~mm}$ lead rod with an energy of $3.0 \mathrm{MeV}$ for the monochromatic beam. The different effects are separated as follows: photo effect, Compton and Rayleigh scattering, annihilation radiation, bremsstrahlung and $E_{\text {total dep. }}$. The vertical line marks the $90^{\circ}$ which divides forward scattering (left-hand side) and back scattering (right-hand side).

ROSI and GEANT4 framework in the format of the polar distribution described in equation 1 . The results show that the most prominent contributions to $E_{\text {total dep. (dark blue) are coherent }}$ and incoherent scattering. In both simulations Rayleigh scattering (light blue) dominates for small angles, while the Compton effect (red) governs the scattered radiation for larger angles in forward direction. Despite the fact that the overall form of the curves in Figure $3 a$ and Figure $3 b$ look qualitatively alike,

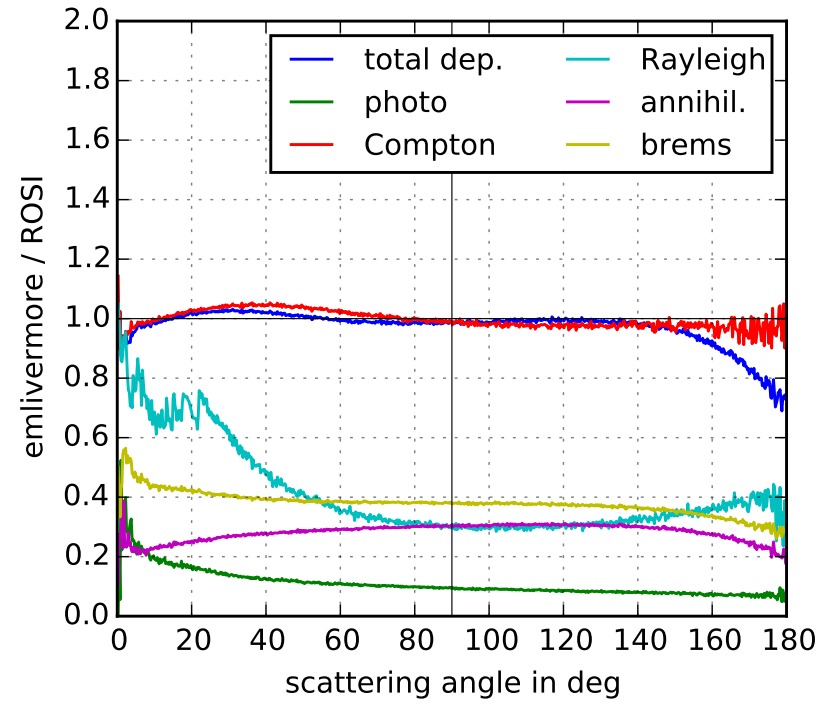

(a) GEANT4 simulation in emlivermore configuration divided by the corresponding ROSI simulation

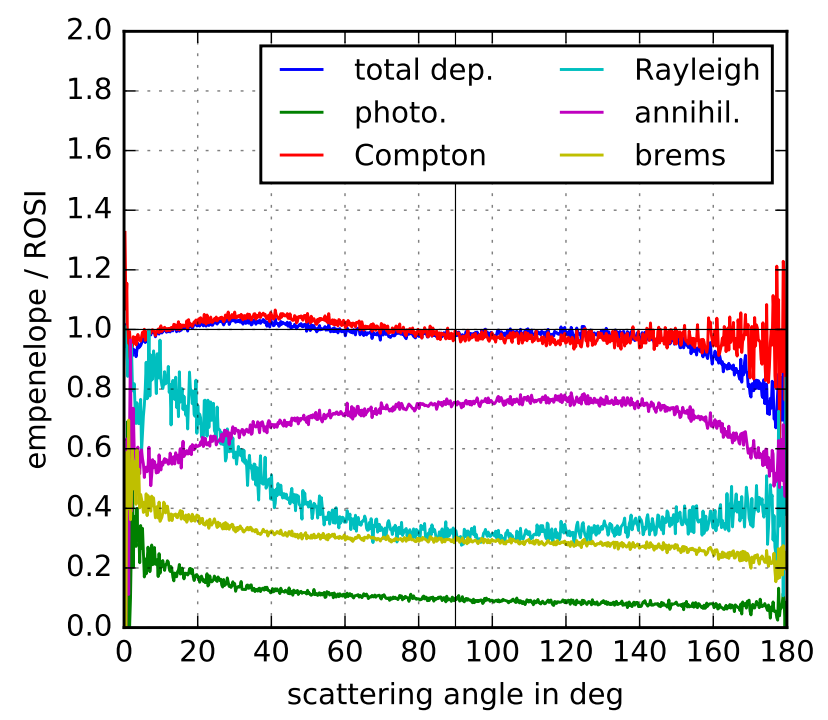

(b) GEANT4 simulation in empenelope configuration divided by the corresponding ROSI simulation

Fig. 4: The setup parameters are kept the same as for Fig. 3 and to guide the eye we added a horizontal line at the the expectation value of a factor of 1 . Both sub-figures $4 \mathrm{a}$ and $4 \mathrm{~b}$ show that total and Compton scattering are in good agreement despite some small variations. The ratio of annihilation radiation is higher in the ROSI simulation. Most differences in the contributing effects can be seen in the photo effect and the bremsstrahlung as well as in the slopes of the Rayleigh scattering.

a closer look reveals discrepancies between the two datasets. While the radial distribution of the remaining effects is almost isotropic in the case of the ROSI simulation results, the data originating from the GEANT4 simulation shows a significant drop of the contribution of the photo effect (dark green) with increasing angles. Another noticeable difference can be seen comparing the contributions of all effects in backwards direction. Not only the overall form of the curves, but also a general 
offset in the deposited energy with respect to those effects can be noticed. While Figure 3a clearly shows that the physical interactions of bremsstrahlung (yellow), annihilation (purple) as well as the photo effect dominate for large angles, the behaviour of the curves in Figure $3 \mathrm{~b}$ certainly paint a profoundly different picture. In fact, for the simulation results originating from the GEANT4 simulation, the Compton interaction dominates up to an angle of $120^{\circ}$, while in the case of the ROSI simulation it was already superseeded as dominating effect by the bremsstrahlung at an angle of about $70^{\circ}$. These differences can be emphasised and subsequently analysed by looking at the ratio of both plots, this is shown in Figure 4a. This direct comparison of emlivermore and ROSI shows a good agreement of the simulation results concerning the total scattering. Only for very large angles in backscattering direction differences up to a factor of two become visible. These differences are accompanied by a large amount of noise in the data set originating from low photon statistics at very large scattering angles. Furthermore, the contribution of Compton interactions is equal for the complete angular range apart from some small variances. The biggest difference occurs in the case of the photo effect. For this effect, the ratio between both simulation results shows that compared to ROSI, emlivermore has a difference in magnitude up to a factor of 0.1 . It is particularly noticeable that while the contributions of the photo effect, the bremsstrahlung and annihilation show a rather constant discrepancy over the whole angular range, the ratio of the contributions of Rayleigh radiation shows a high angular variation. While the results for the Rayleigh effect agree up to $75 \%$ in the range of small angles, this accordance disappears approaching the backwards direction, where the ratio drops down to a factor of 0.3 .

Figure $4 \mathrm{~b}$ shows the corresponding comparison between empenelope and ROSI. Compton and $E_{\text {total dep. }}$ as well as the photo effect show the same agreement as in figure $4 \mathrm{a}$, whereas the annihilation radiation resulting from empenelope is much closer to the ROSI data than the equivalent data from emlivermore. Overall, both of the GEANT4 simulations, compared to the results of the ROSI simulation, show a similar behaviour.

In order to highlight their differences, an additional comparison of these two data-sets has been performed (Fig. 5). Most im-

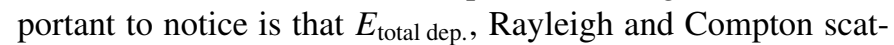
tering as well as the photo effect are equal to each other. Both the remaining effects of bremsstrahlung and annihilation show a rather constant offset, where the bremsstrahlung is about $20 \%$ lower in the empenelope simulation and the annihilation radiation is roughly a factor of 2.4 higher than in the case of emlivermore.

\subsection{Parameter scan - Energy}

For a better overview over the trends of the scattering contributions, we calculated the mean value over all angles to show systematical errors. Moreover, the calculated standard deviation gives an indication on local variations. Figure 6 shows the results for all listed energies in table 2 covering a range of energies from sub-MeV values up to several MeVs. The energy comparison shows large variances for low energies especially for Compton, Rayleigh and bremsstrahlung which decrease at

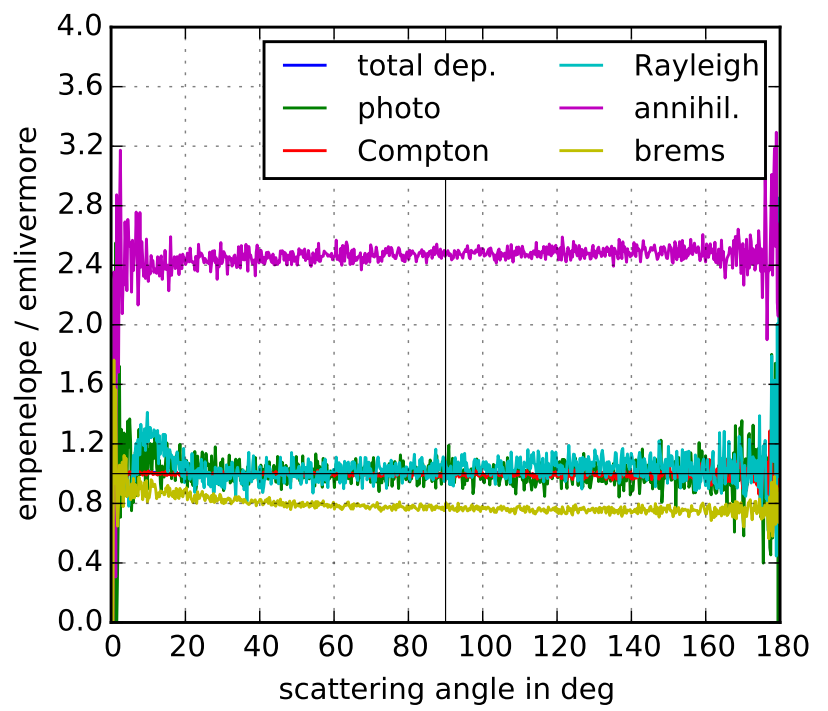

Fig. 5: The simulated data from GEANT4 in the empenelope configuration is here divided by the simulated data from the emlivermore configuration. The process which differs most is the annihilation radiation which is about 2.4 times higher than the one in emlivermore. The next effect which differs is the bremsstrahlung which is higher in the emlivermore simulation. The Rayleigh effect in emlivermore is for angles from $5^{\circ}$ up to $20^{\circ}$ a bit lower for emlivermore, for larger scattering angles it approaches the expected value. The regime of the remaining effects are nearly simulated the same way.

higher energies. With an increasing contribution of Compton interactions at higher energies the ratio of $E_{\text {total dep. }}$ gets closer to unity. In contrast to that, the agreement between the simulation results for Rayleigh scattering decreases with rising energies. At low energies the emlivermore data set shows higher Rayleigh scattering contributions than the data coming from the ROSI simulations, whereas at higher energies this ratio flips and the ratio between the data sets falls drastically below a value of one. The biggest differences with respect to the energy scan can be seen for the photo effect. Overall, the contribution to the photo effect is always lower in the emlivermore simulations. Furthermore, the ratio of the photo effect doesn't show a clear trend in one or the other direction, but appears to change a lot over the range of energies. The difference in annihilation radiation, which is introduced at about $1.1 \mathrm{MeV}$, presents itself in the range of $10 \%$ to $25 \%$. The ratio of the bremsstrahlung shows a rather steep downwards trend with increasing initial energies. From a difference of a factor of 6 at $0.1 \mathrm{MeV}$, it decreases to a value of about one third for energies above $0.4 \mathrm{MeV}$. Looking at Figure 6 in $E_{\text {total dep. }}$ one can see that the mismatch of the contributions is not a constant offset, but changes a lot over the range of energies.

\subsection{Parameter scan - Length}

The length scan was performed at a constant energy of $3.0 \mathrm{MeV}$ and the material was lead (see Figure 7). Excluding the very thin sample of $0.2 \mathrm{~mm}$ length, only slight trends in the ratio for the scattering contributions can be determined. Rayleigh slowly rises with increasing thickness of the material while the annihilation radiation and bremsstrahlung drop slightly. The 


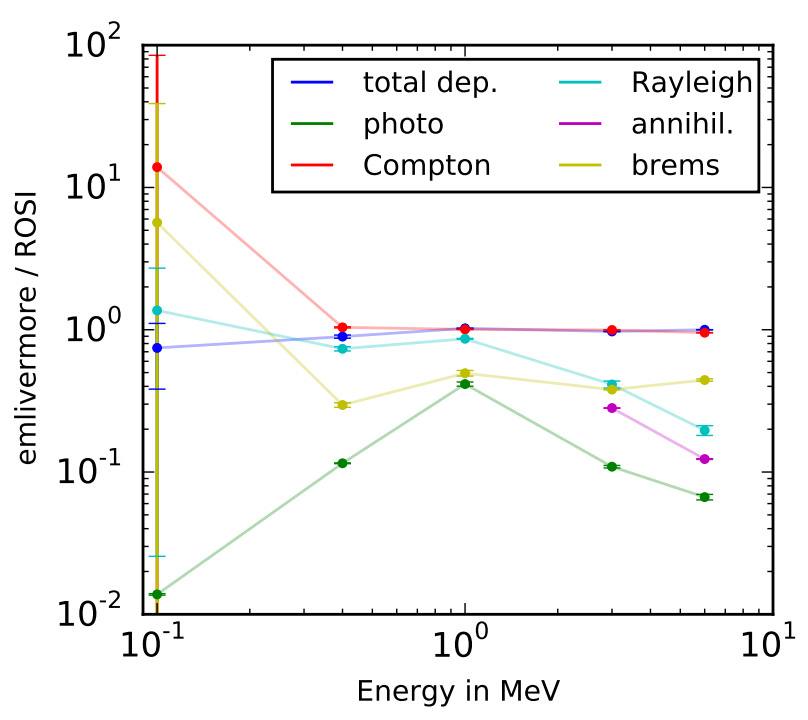

Fig. 6: For a lead rod with the length of $100.0 \mathrm{~mm}$ the factor of emlivermore divided by ROSI with standard deviation vs. the energy is shown.

$E_{\text {total dep. }}$ and Compton scattering are in good agreement over all lengths. For the thinnest object, at a length of $0.2 \mathrm{~mm}$, all effects show large standard deviations compared to the values at larger lengths. Most notable are the extremely large standard deviations in the case of Rayleigh and annihilation radiation.

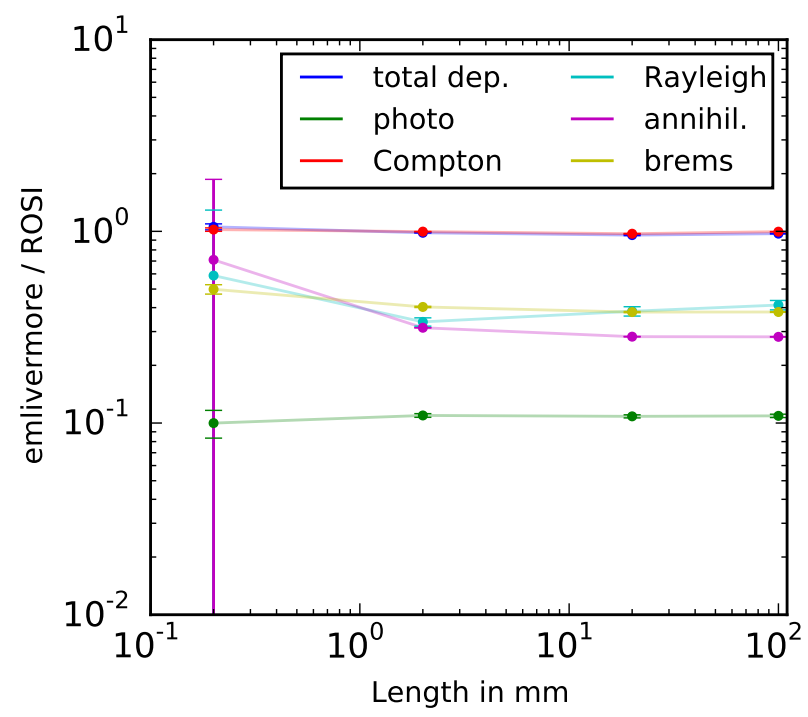

Fig. 7: For a lead rod at an photon energy of $3.0 \mathrm{MeV}$ the factor of emlivermore divided by ROSI with standard deviation vs. the length is shown.

\subsection{Parameter scan - Material}

Finally, the influence that the atomic number of the material had on the difference in the simulation results was investigated. Figure 8 shows the various materials of different density, starting from low to high, there are PVC $\left(\left[\mathrm{C}_{2} \mathrm{H}_{3} \mathrm{Cl}\right]_{\mathrm{n}}\right)$, aluminium, steel $(\mathrm{FeC})$ and lead. The rod length at $100.0 \mathrm{~mm}$ and the energy at 3.0 MeV were kept constant.
For PVC (Fig. 8a) $E_{\text {total dep. }}$ and Compton scattering are in very good agreement except for a spike in the curve corresponding to $E_{\text {total dep. }}$ at angles less than $1^{\circ}$. Obviously, the Rayleigh scattering shows the largest differences between the two data sets. Moreover, the ratio concerning Rayleigh scattering is noisy. The annihilation radiation is nearly isotropic and the ratio of the emlivermore simulation reaches about $35 \%$ of the value originating from the ROSI simulation. The photo effect is nearly invisible because it is about 100 times smaller on emlivermore. The ratio of bremsstrahlung drops from about $90 \%$ in forward scattering to about $30 \%$ in backscattering.

Aluminium (see Fig. 8b) shows a similar behaviour like the PVC contributions but the spike in $E_{\text {total dep. }}$ broadened and increased. The annihilation ratio went up to about $40 \%$ while the bremsstrahlung ratio dropped to $85 \%$ in forward scattering direction. As in the previous example of PVC, the Rayleigh scattering presents itself with a lot of noise and big variations. The mean value of the ratio of Rayleigh scattering is 22 and its standard deviation is 57720 . The photo effect, with a contribution of about $0.03 \%$ is even smaller than in the previous simulation.

Steel (see Fig. 8c) is similar to the already described lead sample (see Fig. 8d). It shows a drop in the $E_{\text {total dep. for large an- }}$ gles (above $160^{\circ}$ ), while the Compton scattering shows a good agreement. The Rayleigh scattering in the small angle regime is in better agreement than in the previous examples of low $\mathrm{Z}$ materials. Moreover, it became less noisy, however, both data sets are still not in good agreement for larger angles, where the ratio of Rayleigh scattering contributions drops down to $25 \%$. The ratio of the annihilation radiation is about $50 \%$ higher than in every other material. The photo effect shows the same behaviour as aluminium. The ratio concerning bremsstrahlung shows a drop over the angular range as it has already been observed in the lighter materials. Here the agreement is worse, the ratio drops from a value of $60 \%$ down to $25 \%$ in backwards direction. It is worth to notice that with increasing atomic number, the different effects increase or decrease with respect to each other, while the overall trend of the ratios does not seem to change. The ratio describing the contribution of bremsstrahlung for example shows a downward trend with increasing angle for all material configurations. However, the agreement, especially in the forward direction drops considerably with increasing density. The agreement concerning the Rayleigh scattering however increases with higher $\mathrm{Z}$.

\section{Discussion}

Overall, the results show a good agreement of both codes regarding $E_{\text {total dep. }}$ as well as Compton scattering. Considering the high discrepancies between the physical effects, which are highlighted in Figure 4, this accordance of the results with respect to the total deposited energy seems strange. However, the reason for this inconsistency lies in the inherent dominance of the Compton interaction over all other physical interactions in the considered energy regime. The prevalence of the Compton interaction hides the fact that all other physical interactions show a strong disagreement, making it invisible to a user who 


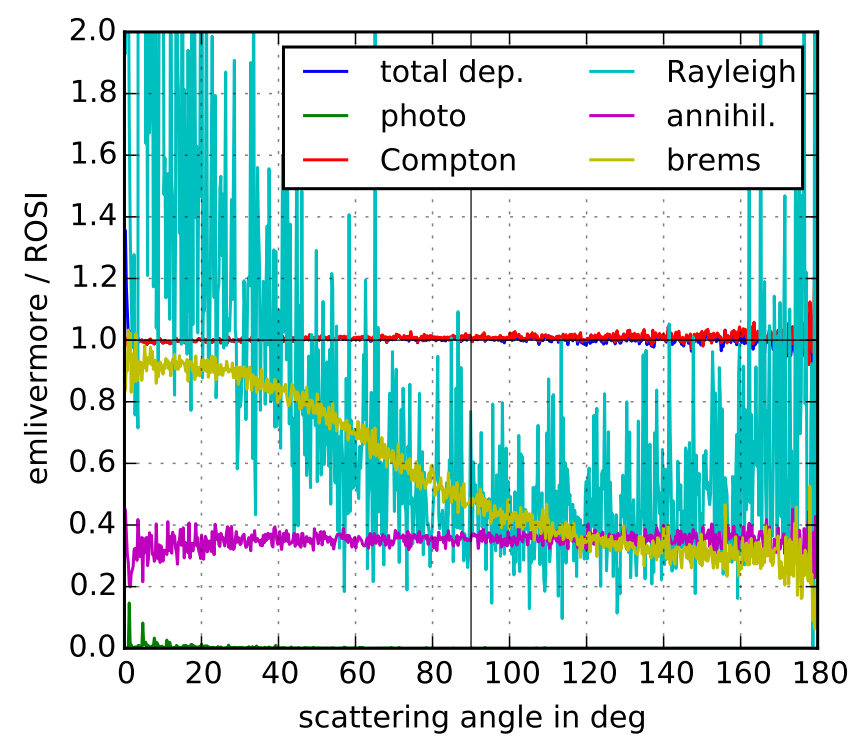

(a) PVC

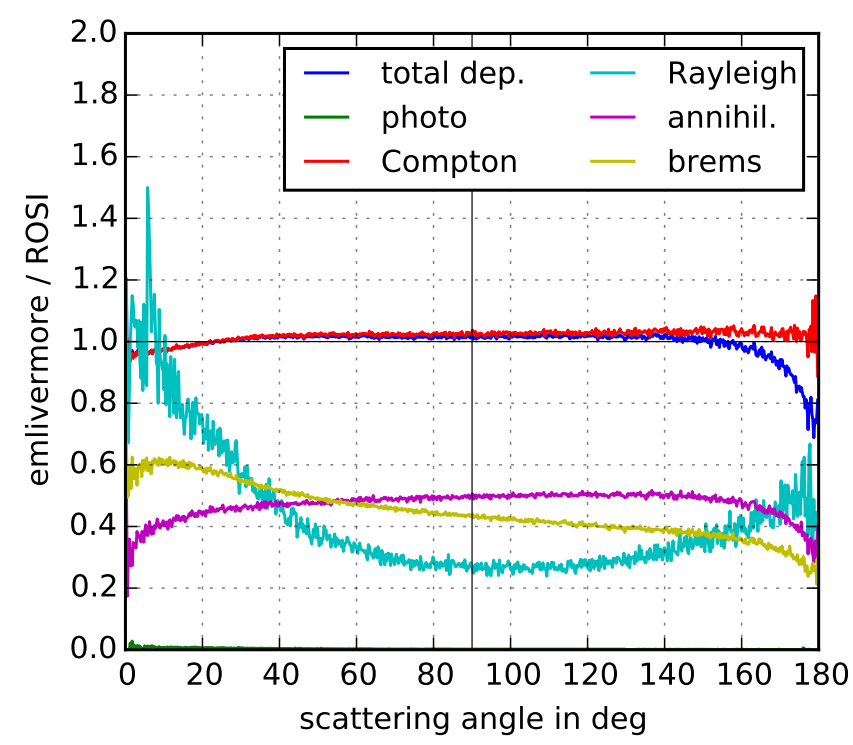

(c) $\mathrm{FeC}$

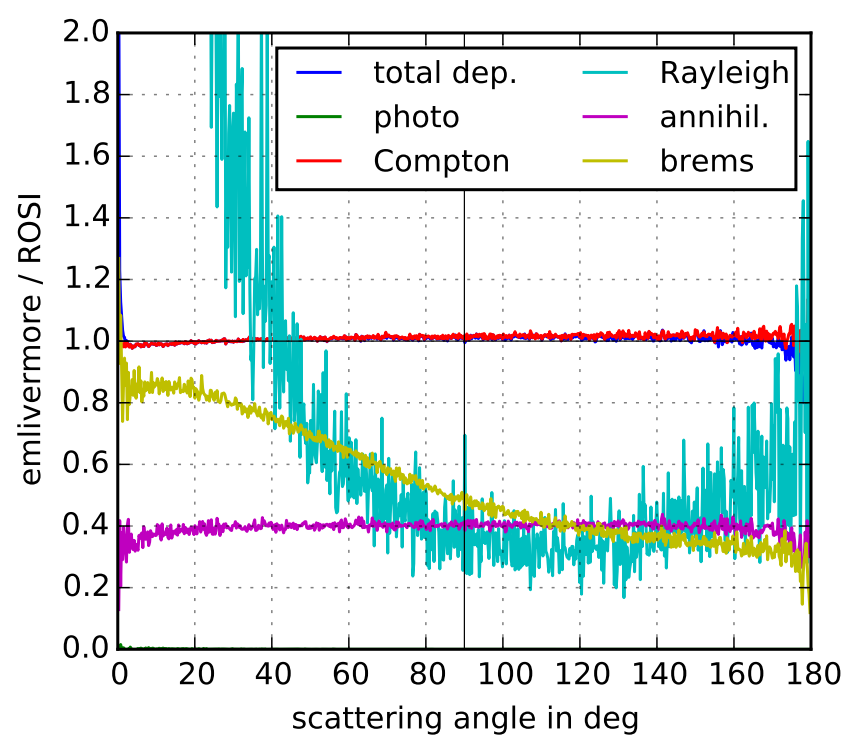

(b) $\mathrm{Al}$

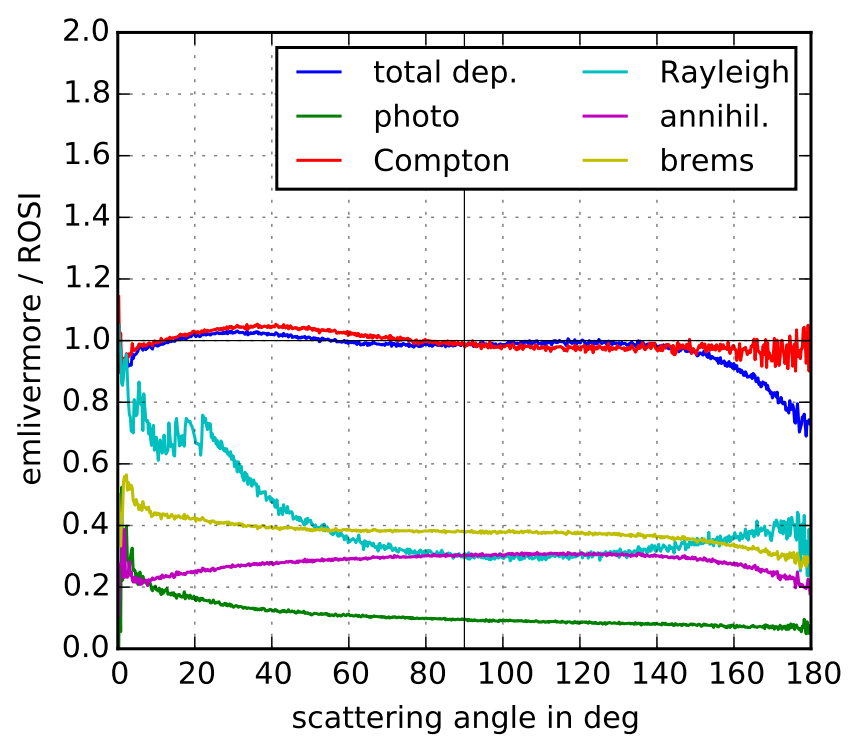

(d) $\mathrm{Pb}$

Fig. 8: Comparison for different materials form PVC 8 a over aluminium $8 \mathrm{~b}$ and steel $8 \mathrm{c}$ to lead $8 \mathrm{~d}$. For all simulations the energy was kept constant at $3.0 \mathrm{MeV}$ and the length was set to $100.0 \mathrm{~mm}$.

is only checking the overall signal resulting from the simulation. This means that the dominance of the Compton interaction can explain the good agreement of the simulation results; nonetheless, it also raises further questions regarding the differences in agreement for all of the physical effects that are being examined in this work. It is not obvious why the two simulation frameworks would produce similar results concerning one type of interaction, while the agreement seems to fail with respect to all other interactions. First of all it is interesting to notice that the discrepancies at hand not only show discrepancies in the corresponding amount of their contribution, but also in their polar distribution. These differences occur at the whole energy range (compare Figure 6). Moreover, even in the case of a comparison between the two GEANT4 configurations differences in contribution can be noticed. This last fact that not only the results of the ROSI simulation with respect to the ones of the GEANT4 simulations (compare Figure 5), but also the results between the two GEANT4 simulations using emlivermore and empenelope (compare Figure 5) show differences is a first clue that the discrepancies might stem from differences in the cross sections used for the simulation. A closer look at table 1 emphasises this inkling. It can be seen that, apart from the Compton interaction, the physical models used for the cross sections differ greatly. Some use analytical formulas with or 
without correction terms, while others use parameterization of measured data points to generate the cross sectional data. This does not only explain the discrepancies as seen in Figure 4 for example, but in fact also explains the high agreement concerning the Compton interaction and as a results of course also the accordance with respect to the total deposited energy.

A more in depth analysis of the discrepancies concerning their angular distribution as well as their overall amount shows that the highest variation seems to occur for the effect of Rayleigh scattering (compare Figure 5). It seems like the agreement changes particularly in forward and backward direction in this case. However, this cannot be attributed to differences in the particle transport code as well as the cross sectional data. In fact, the variation over the angular range that can be seen in this case is accompanied by high standard deviations which stem from low photon statistics. This means that a reliable interpretation concerning the form and quantity of the ratio of Rayleigh scattering is not possible.

In general, statements on the distribution of physical interactions are less meaningful in backward than in forward direction. Due to low counts in detector elements, the statistics are not reliable for both very small angles in forward direction as well as very large angles. This leads to a decrease in accuracy and in addition to a reduced comparability of both data-sets for those angles.

The biggest overall difference concerning the amount of scattered radiation can be seen for the photo effect. This discrepancy can be ascribed to the fact that in contrast to ROSI, the GEANT4 simulations did not incorporate fluorescence. The remaining discrepancy of accordance between physical interactions of all considered simulation codes can be attributed to the aforementioned differences in the implemented cross sections. The physical models of the libraries used for the calculation of the interaction probabilities seem to lead to significantly different results.

Another clue for this conclusion can be found in the parameter sweeps shown in Figures 6, 7 and 8. A scan over the parameter energy revealed that differences in the simulation results do not show a systematic behaviour. In fact, the deviations, especially in the case of the photo effect, show a rather random behaviour throughout the energy range instead of a general offset of the contributions. In contrast to this, the length scan shows very little variation in the ratio of the data sets. Only the thinnest object at a length of $0.2 \mathrm{~mm}$ shows a large deviation to the other values along the length scan. This is due to the large decrease in statistics for the scatter-events in this case. For a thin object, the same number of simulated events will produce a considerably smaller amount of scattered photons than a thicker object. This means that for the thinner object, most simulated photons will end up in the primary beam, which is not considered in the evaluation. For the case of a lead object measured with an energy of $3 \mathrm{MeV}$, the total number of scattered events will decrease by a factor of 100 from the $100 \mathrm{~mm}$ object to the $0.2 \mathrm{~mm}$ object. This difference in behaviour in direction of energy and length over the parameter space is another indication that the main reason for differences in simulation results is the data base for cross sections and not the overall particle transport mechanics of the simulations. It can be explained by the fact that the sampled cross sections of a simulation depend on the energy of the particles and are not influenced by the length of the objects.

While the simulation results are clearly not influenced by a varying length of an investigated object (compare Fig. 7), the material of the object is expected to have a just as big influence on the simulation results as the energy. This is due to the fact that cross sections are not only dependent on energy, but also on the atomic number of the material, which is of course true for single materials as well as composites. The scan over different materials confirms this assumption (compare Fig. 8). It can be seen, that the polar distribution of the scattered radiation very much depends on the density of the measured materials. For low $\mathrm{Z}$ materials for example, we noticed that the dip for high scattering angles is not as broad and deep compared to dense materials. Moreover, with increasing density, the Rayleigh scattering gets closer to the optimal factor of 1 in the regime of small angles. This means, not only the simulated energies, but also the materials have a noticeable impact on simulation results. As a result, the modelling of the simulated materials has to be performed with care.

Overall, in the case of the energy regime considered here, the results imply that the general results of both frameworks will match in most cases, whereas the individual contributions of the physical effects will always show big discrepancies.

\section{Conclusion}

In this work, two popular MC simulation frameworks have been compared in the context of X-ray CT setups. The comparison was performed with the help of a parameter study considering variations in the energy of the X-ray beam, the material of the sample as well as the length of the sample.

While the total deposited energy as well as the contribution of Compton scattering show a good agreement between all simulated configurations, all other physical effects exhibit large deviations in a comparison of data-sets. It has been shown, that this good agreement of the total simulated signal as well as the high discrepancies concerning the other physical interactions of Rayleigh scattering, photo effect, annihilation and bremsstrahlung stem from the differences in the databases used for the cross sectional data. The agreement of results concerning the Compton interaction, which used the same physical model for sampling the cross sectional data in all database realisations, shows that the particle transport mechanics of both simulation frameworks produce the same results. A scan over energy, length and material shows that the two parameters, energy and material, have a significant influence on the agreement of the simulation results, while the length parameter shows no noticeable influence on the deviations between the data-sets. The results of this work imply that, in the context of X-Ray physics as shown in this work, the overall simulated signals resulting from both frameworks will match. However, an investigation of the results concerning individual contributions of physical interactions is not recommendable. 
Overall, the results show that the databases for low energy physics form a crucial element of X-ray simulations, which has to be chosen with care before performing simulations. This is even more important for tasks that need spectroscopic information. A more detailed discussion of the topic could be done on spectral simulation results, which will highlight the differences between the simulations in a more detailed manner. Moreover, a discussion of multiple scattering has not been performed in this work. Especially a closer look at the Compton effect with respect to single and multiple scattering could be interesting in a next step.

\section{Acknowledgement}

This work is part of the project " $\mathrm{X}$-ray 3D Computed Tomography with Mega Electronvolt Source" (CTOMES). The authors would like to thank the German Research Foundation (DFG) and the Swiss National Science Foundation (SNF) for funding.

\section{References}

[1] J. Allison, K. Amako, J. Apostolakis, H. Araujo, P. A. Dubois, M. Asai, G. Barrand, R. Capra, S. Chauvie, R. Chytracek, et al., Geant4 developments and applications, Nuclear Science, IEEE Transactions on 53 (1) (2006) 270-278.

[2] A. Miceli, R. Thierry, A. Flisch, U. Sennhauser, F. Casali, M. Simon, Monte carlo simulations of a high-resolution X-ray ct system for industrial applications, Nuclear Instruments and Methods in Physics Research Section A: Accelerators, Spectrometers, Detectors and Associated Equipment 583 (2) (2007) 313-323.

[3] N. Freud, J.-M. Létang, D. Babot, A hybrid approach to simulate multiple photon scattering in X-ray imaging, Nuclear Instruments and Methods in Physics Research Section B: Beam Interactions with Materials and Atoms 227 (4) (2005) 551-558. doi:10.1016/j.nimb.2004.10.001.

[4] A. Badano, J. Sempau, MANTIS: combined x-ray, electron and optical Monte Carlo simulations of indirect radiation imaging systems., Physics in Medicine and Biology 51 (2006) 1545-1561. doi:10.1088/00319155/51/6/013.

[5] A.-P. Colijn, F. J. Beekman, Accelerated simulation of cone beam x-ray scatter projections, Medical Imaging, IEEE Transactions on 23 (5) (2004) 584-590.

[6] Y. Kyriakou, T. Riedel, W. A. Kalender, Combining deterministic and monte carlo calculations for fast estimation of scatter intensities in ct, Physics in medicine and biology 51 (18) (2006) 4567.

[7] R. Thierry, A. Miceli, J. Hofmann, A. Flisch, U. Sennhauser, Hybrid simulation of scatter intensity in industrial cone-beam computed tomography, Nuclear Instruments and Methods in Physics Research Section A: Accelerators, Spectrometers, Detectors and Associated Equipment 598 (2) (2009) 611-619.

[8] J. Giersch, A. Weidemann, G. Anton, ROSI-an object-oriented and parallel-computing Monte Carlo simulation for X-ray imaging, Nuclear Instruments and Methods in Physics Research Section A: Accelerators, Spectrometers, Detectors and Associated Equipment 509 (1-3) (2003) 151-156. doi:10.1016/S0168-9002(03)01564-X.

[9] S. Agostinelli, et al., GEANT4 - A simulation toolkit, Nuclear Instruments and Methods in Physics Research, Section A: Accelerators, Spectrometers, Detectors and Associated Equipment 506 (3) (2003) 250-303. doi:10.1016/S0168-9002(03)01368-8.

[10] H. Zaidi, M. R. Ay, Current status and new horizons in monte carlo simulation of x-ray ct scanners, Medical \& biological engineering \& computing 45 (9) (2007) 809-817.

[11] J. Giersch, D. Niederlöhner, G. Anton, The influence of energy weighting on X-ray imaging quality, Nuclear Instruments and Methods in Physics Research Section A: Accelerators, Spectrometers, Detectors and Associated Equipment 531 (1) (2004) 68-74.
[12] S. Pistrui-Maximean, N. Freud, J. Létang, A. Koch, B. Munier, A. Walenta, G. Montarou, D. Babot, Geant 4 simulation of the response of phosphor screens for $\mathrm{X}$-ray imaging, Nuclear Instruments and Methods in Physics Research Section A: Accelerators, Spectrometers, Detectors and Associated Equipment 563 (1) (2006) 196-199.

[13] A. Loehr, J. Durst, T. Michel, G. Anton, P. Geithner, Comparison of recent experimental data with Monte Carlo tools such as RoSi, Geant4 and Penelope, IEEE Nuclear Science Symposium Conference Record (2009) 3904-3908doi:10.1109/NSSMIC.2009.5401931.

[14] J. Giersch, A. Weidemann, Lscat-gismo. an object-oriented framework for particle simulation, Proceedings of the third international workshop on EGS.

[15] Y. Namito, H. Hirayama, LSCAT: Low-Energy Photon-Scattering Expansion for the EGS4 Code (Inclusion of Electron Impact Ionization), KEK Internal 95-10.

[16] R. D. W. O. Bielajew, A. F., W. R. Nelson, H. Hirayama, History, overview and recent improvements of EGS4, in: Presented at, no. SLACPUB-6499, 1994.

[17] S. L. A. C. C. Group, A. Cook, A User's Guide to MORTRAN. A User's Guide to CDC 7600/6600 MORTRAN., 1973.

[18] D. E. Cullen, J. H. Hubbell, L. Kissel, EPDL97: The evaluated photon data library '97', UCRL-LR-50400 Vol 6 Rev 56 (1997) 1-35.

[19] M. J. Berger, J. H. Hubbell, S. M. Seltzer, J. Chang, J. S. Coursey, R. Sukumar, D. S. Zucker, K. Olsen, Xcom: Photon cross sections database, NIST Standard reference database 8.

[20] S. Perkins, D. Cullen, M. Chen, J. Rathkopf, J. L. L. N. L. Scofield, J. N. I. o. S. Hubbell, Technology, Tables and graphs of atomic subshell and relaxation data derived from the LLNL Evaluated Atomic Data Library (EADL), Z = 1-100, 1991.

[21] S. Perkins, D. L. L. N. L. Cullen, S. N. I. o. S. Seltzer, T. (NML), Tables and graphs of electron-interaction cross sections from $10 \mathrm{eV}$ to $100 \mathrm{GeV}$ derived from the LLNL Evaluated Electron Data Library (EEDL), Z = $1-100,1991$.

[22] L. Storm, H. I. Israel, Photon cross sections from $1 \mathrm{keV}$ to $100 \mathrm{MeV}$ for elements $Z=1$ to $Z=100$, Atomic Data and Nuclear Data Tables 7 (6) (1970) 565-681. doi:10.1016/S0092-640X(70)80017-1.

[23] J. H. Hubbell, I. $\varnothing$ verb $\varnothing$, Relativistic atomic form factors and photon coherent scattering cross sections (1979). doi:doi:10.1063/1.555593.

[24] J. W. Motz, H. a. Olsen, H. W. Koch, Pair production by photons, Reviews of Modern Physics 41 (4) (1969) 581-639. doi:10.1103/RevModPhys.41.581.

[25] J. C. Butcher, H. Messel, Electron number distribution in electron-photon showers in air and aluminium absorbers, Nuclear Physics 20 (1960) (1960) 15-128. doi:10.1016/0029-5582(60)90162-0.

[26] H. W. Koch, J. W. Motz, Bremsstrahlung Cross-Section Formulas and Related Data, Reviews of Modern Physics 31 (4) (1959) 920-955. doi:10.1103/RevModPhys.31.920.

[27] H. Kolbenstvedt, Simple theory for K-ionization by relativistic electrons, Journal of Applied Physics 38 (12) (1967) 4785-4787. doi:10.1063/1.1709220.

[28] M. Kiunke, R. Schielein, K. Dremel, S. Zabler, F. Sukowski, S. Kasperl, Monte Carlo X-ray scattering studies in the $\mathrm{MeV}$ regime, Proceeding Eleventh European Conference on Non-Destructive Testing (11th ECNDT). 